A Fast Semi-Inverse Approach to Detect and Remove the Haze from a Single Image

Non Peer-reviewed author version

ANCUTI, Codruta; ANCUTI, Cosmin; HERMANS, Chris \& BEKAERT, Philippe

(2011) A Fast Semi-Inverse Approach to Detect and Remove the Haze from a Single Image. In: COMPUTER VISION - ACCV 2010, Pt II.p. 501-514.

Handle: http://hdl.handle.net/1942/11702 


\title{
A Fast Semi-Inverse Approach to Detect and Remove the Haze from a Single Image
}

\author{
Codruta O. Ancuti, Cosmin Ancuti, Chris Hermans, Philippe Bekaert \\ Hasselt University - tUL -IBBT, \\ Expertise Center for Digital Media, Wetenschapspark 2, Diepenbeek, 3590, Belgium
}

\begin{abstract}
In this paper we introduce a novel approach to restore a single image degraded by atmospheric phenomena such as fog or haze. The presented algorithm allows for fast identification of hazy regions of an image, without making use of expensive optimization and refinement procedures. By applying a single per pixel operation on the original image, we produce a 'semi-inverse' of the image. Based on the hue disparity between the original image and its semi-inverse, we are then able to identify hazy regions on a per pixel basis. This enables for a simple estimation of the airlight constant and the transmission map. Our approach is based on an extensive study on a large data set of images, and validated based on a metric that measures the contrast but also the structural changes. The algorithm is straightforward and performs faster than existing strategies while yielding comparative and even better results. We also provide a comparative evaluation against other recent single image dehazing methods, demonstrating the efficiency and utility of our approach.
\end{abstract}

\section{Introduction}

In outdoor environments, light reflected from object surfaces is commonly scattered due to the impurities of the aerosol, or the presence of atmospheric phenomena such as fog and haze. Aside from scattering, the absorption coefficient presents another important factor that attenuates the reflected light of distant objects reaching the camera lens. As a result, images taken in bad weather conditions (or similarly, underwater and aerial photographs) are characterized by poor contrast, lower saturation and additional noise.

Image processing applications commonly assume a relatively transparent transmission medium, unaltered by the atmospheric conditions. Outdoor vision applications such as surveillance systems, intelligent vehicles, satellite imaging, or outdoor object recognition systems need optimal visibility conditions in order to detect and process extracted features in a reliable fashion. Since haze degradation effects depend on the distance, as disclosed by previous studies [1, 2] and observed as well in our experiments (see Fig. 1), standard contrast enhancement filters such as histogram stretching and equalization, linear mapping, or gamma correction are limited to perform the required task introducing halos artifacts and distorting the color. The contrast degradation of a hazy image 

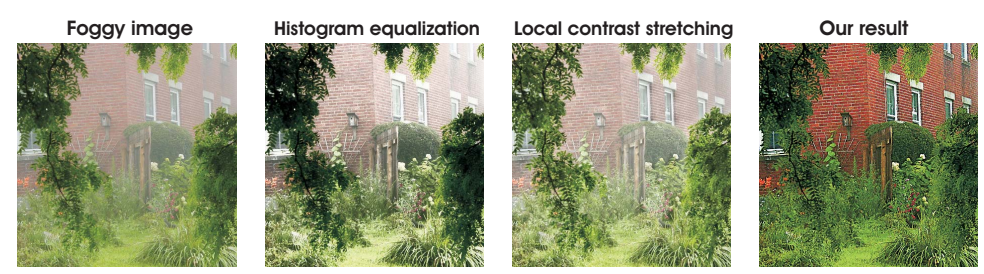

Fig. 1. Standard techniques limitations. From left to right: initial foggy images, histogram equalization, local contrast stretching and our restored result.

is both multiplicative and additive. Practically, the haze effect is described by two unknown components: the airlight contribution and the direct attenuation related to the surface radiance. The color ambiguity of the radiance is due to the additive airlight, which increases exponentially with the distance. Enhancing the visibility of such images is not a trivial task, as it poses an inherently underconstrained problem. A reliable restoration requires an accurate estimation of both the true colors of the scene and the transmission map, closely related to the depth-map.

Recently, there has been an increased interest in the vision and graphics communities in dehazing single images [1-5]. In this paper we introduce an alternative approach to solve this challenging problem. Our technique is based on the remark that the distance from the observer to the scene objects is highly correlated with the contrast degradation and the fading of the object colors. More specifically, by an extensive study it has been disclosed an important difference between hazy and non-hazy image regions, by performing a per pixel comparison of the hue values in the original image to their values in a 'semi-inversed' image. This 'semi-inversed' image version is obtained by replacing the RGB values of each pixel on a per channel basis by the maximum of the initial channel value $(r, g$ or $b)$ and its inverse $(1-r, 1-g$ or $1-b)$, followed by an image-wide renormalization. This observation has been validated on a large set of images, and allows for the detection of the hazy image regions by applying only a single simple operator. This facilitates the estimation of the airlight constant color, and enables us to compute a good approximation of the haze-free image using a layer-based approach.

Contributions. This paper introduces the following three main contributions: - first of all, we introduce a novel single image algorithm for the automatic detection of hazy regions.

- secondly, our approach works on a per pixel basis. This makes it suitable for parallelization, and allows us to retain sharp detail near edges.

- finally, our layer-based fusion dehazing strategy yields comparative and even better restored results than the existing approaches but performs faster being suitable for real-time applications. 


\section{Related Work}

An important area of applications for dehazing algorithms can be found in multispectral remote sensing applications, where specialized sensors installed on satellites capture a specific band of the reflected light spectrum. Due to aerosol impurities and cloud obstruction, the recorded images require specific processing techniques $[6,7]$ to recover the original information.

Many haze-removal techniques have used additional information in order to facilitate the search for a solution to this underconstrained problem. Some methods $[8,9]$ employ multiple images of the same scene, taken under various atmospheric conditions or combined with near-infrared version [10]. Polarization methods [11-13] exploit the fact that airlight is partially polarized. By taking the difference of two images of the same scene under different polarization angles, it becomes possible to estimate the magnitude of the polarized haze light component. Another category of techniques assume a known model of the scene. Narasimhan and Nayar [14] employ a rough approximated depth-map obtained by user assistance. Deep Photo [15] uses the existing georeferenced digital terrain and urban models to restore foggy images. By using an iterative registration method, they align the 3D model with the outdoor images, producing the depth map required by the restoration process.

Recently, several single image based methods [1-5] have been introduced. The method of Fattal [1] uses a graphical model that solves the ambiguity of airlight color based upon the assumption that image shading and scene transmission are locally uncorrelated. The approach of [5] proposed a related method with [1] solution. This method models the image with a factorial MRF and computes the albedo and depth independently like two statistically independent latent layers. In Tan's approach [2], the restoration aims to maximize the local contrast. He et al. [3] employ the dark channel image prior, based on statistical observation of haze-free outdoor images, in order to generate a rough estimation of the transmission map. Subsequently, due to the fact that they approximate the scene using patches of a fixed size, a matting strategy is required in order to extrapolate the value into unknown regions, and refine the depth-map. Tarel and Hautiere [4] introduced a contrast-based enhancing approach to remove the haze effects, aimed at being faster than the previous approaches.

\section{The Optical Model}

The optical model used in this paper is similar to the one employed in previous single image dehazing methods [1-4], initially described by Koschmieder [16]. For the sake of completeness, a brief description of this model is presented in the following section.

When examining an outdoor scene from an elevated position, features gradually appear to become lighter and fading as they are closer towards the horizon. Only a percentage of the reflected light reaches the observer as a result of the absorption in the atmosphere. Furthermore, this light gets mixed with the airlight [16] color vector, and due to the scattering effects the scene color is 


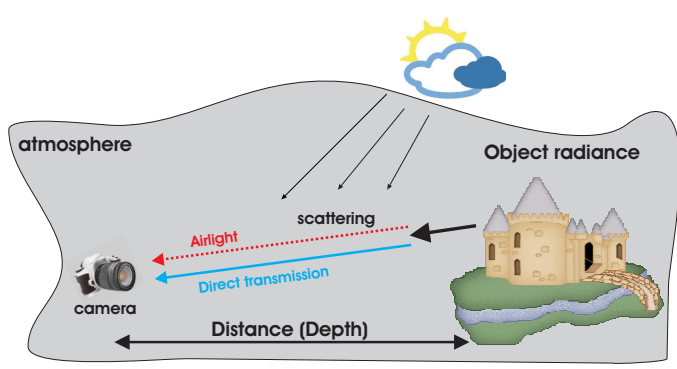

Fig. 2. Optical model.

shifted (illustrated in Fig. 2). Based on this observation, the captured image of a hazy scene $\mathcal{I}_{h}$ is represented by a linear combination of direct attenuation $\mathcal{D}$ and airlight $\mathcal{A}$ contributions:

$$
\mathcal{I}_{h}=\mathcal{D}+\mathcal{A}=\mathcal{I} * t(x)+A_{\infty} *(1-t(x))
$$

where $\mathcal{I}_{h}$ is the image degraded by haze, $\mathcal{I}$ is the scene radiance or hazefree image, $A_{\infty}$ is the constant airlight color vector and $t$ is the transmission along the cone of vision. This problem is clearly ill-posed, and requires us to recover the unknowns $\mathcal{I}, A_{\infty}$ and $t(x)$ from only a single input image $\mathcal{I}_{h}$. In a homogeneous atmosphere, the transmission $t$ is considered to be modulated as:

$$
t(x)=\exp (-\beta * d(x))
$$

where $\beta$ is the attenuation coefficient of the atmosphere due to the scattering and $d$ represents the distance to the observer.

From equation 1, it becomes apparent that the chrominance attenuation becomes increasingly influenced by the airlight, as the optical depth increases:

$$
\frac{\mathcal{A}}{\mathcal{D}}=\frac{A_{\infty} *(1-t(x))}{\mathcal{I} * t(x)}
$$

Theoretically, if the transmission and the airlight are known the haze-free image can be easily computed:

$$
\mathcal{I}=A_{\infty}-\left(A_{\infty}-\mathcal{I}_{h}\right) / t(x)
$$

\section{Haze Detection}

The dark object method [6] is a well-known technique within the remote sensing community, where it is employed to remove haze from homogeneous scenes. More recently, He et al. [3] have presented a new derivation of this approach, called the dark channel strategy. A disadvantage of this new method is its inability to properly preserve edges, which is caused mainly by the employed erosion filter during the stage of computing the dark channel. In order to recover the refined 

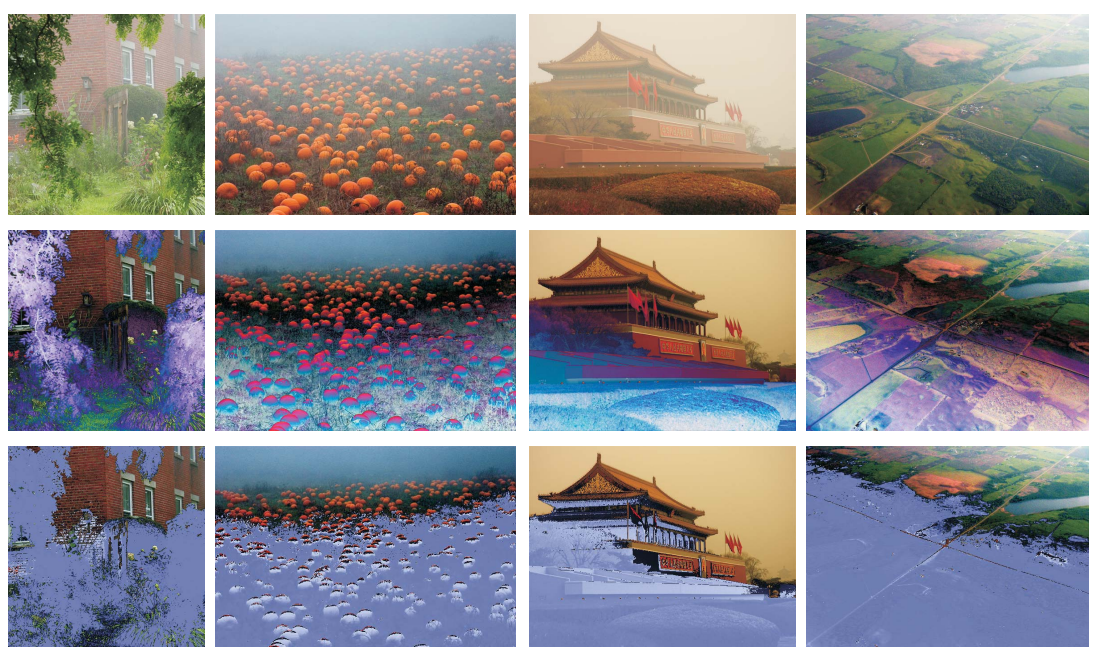

Fig. 3. Haze detection. The first row shows the original hazy images $\mathcal{I}$. In the second row, we show the yielded semi-inversed image $\mathcal{I}_{s i}$. Finally, in the third row, we label the pixels identified as not under the influence of haze with a blue mask. In these regions, the intensity of the blue color is proportional with the hue disparity.

transmission map and the latent image, this patch-based approach requires a complex postprocessing stage. By employing the dark channel prior [3], it has been shown that each patch of a natural image contains at least one point that is dark for non-sky or haze-free regions. The validity of this observation is mainly motivated by the fact that natural images are colorful and full of shadows [3]).

In this work we introduce a novel per pixel method that aims at generalizing the previous dark-channel approach. During our experiments, in which we analyzed a large set of natural images degraded by haze, we have observed that in haze-free and non-sky images, pixels in the neighborhood of dark pixels have a low intensity value in at least one color channel $(r, g$ or $b)$. On the dark channel, patches representing sky and hazy regions contain high values, as the local minimal intensity of such patches is high. Similarly, it has been observed that pixels in sky or hazy areas have high values in all color channels. These observations confirm the assumption that values in hazy image patches vary smoothly, except at depth discontinuities.

Based on these observations, we introduce a direct haze detection algorithm that operates in a pixel-wise manner. We create a semi-inversed image $\mathcal{I}_{s i}(x)=$ $\left[\mathcal{I}_{s i}^{r}, \mathcal{I}_{s i}^{g}, \mathcal{I}_{s i}^{b}\right]$. This image can be obtained by replacing the RGB values of each pixel $x$ on a per channel basis by the maximum of the initial channel value and 

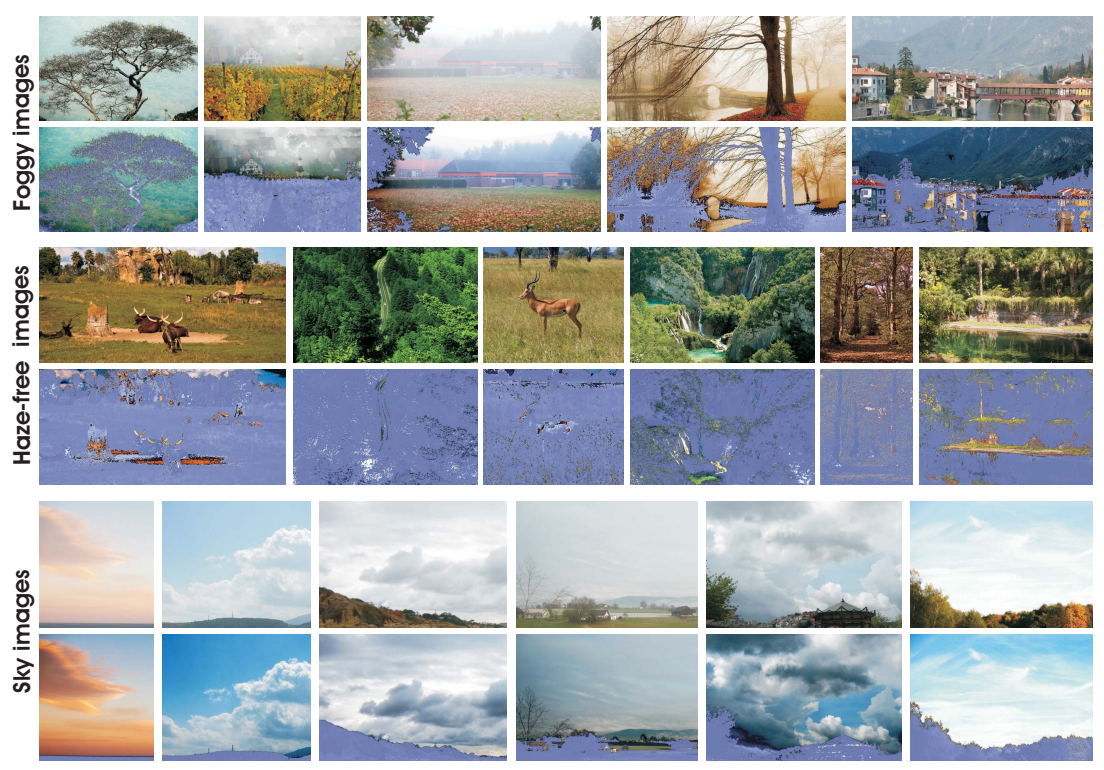

Fig. 4. Results from applying our haze detection procedure on a large data set of images. Overall, haze-free images contain 96\% pixels labeled as haze-free (masked in blue), while hazy and sky images are characterised by a significant decrease in haze-free pixels (less than 13\%).

its inverse:

$$
\begin{aligned}
& \mathcal{I}_{s i}^{r}(x)=\max _{x \in \mathcal{I}}\left[\mathcal{I}^{r}(x), 1-\mathcal{I}^{r}(x)\right] \\
& \mathcal{I}_{s i}^{g}(x)=\max _{x \in \mathcal{I}}\left[\mathcal{I}^{g}(x), 1-\mathcal{I}^{g}(x)\right] \\
& \mathcal{I}_{s i}^{b}(x)=\max _{x \in \mathcal{I}}\left[\mathcal{I}^{b}(x), 1-\mathcal{I}^{b}(x)\right]
\end{aligned}
$$

where $\mathcal{I}^{r}(x), \mathcal{I}^{g}(x)$ and $\mathcal{I}^{b}(x)$ represent the $R G B$ channels of a considered image pixel $x$. Because the operations performed in equation 5 map the range of all pixels of the semi-inversed image $\mathcal{I}_{s i}$ onto the range $[0.5,1]$, renormalization is required.

The reason of hue disparity is due to the image characteristics that have been previously described. In haze-free areas since at least one-channel is characterized by small values the operation will replace that value with its inverse. In regions of sky or haze since all values are characterized by high values, the max operation will return the same values. Therefore, by this direct hue comparison of the semiinverse with the original image version, we are able to find pixels that need to be restored while conserving a similar color appearance with the original one.

As illustrated in Fig. 3, this simple operation produces a semi-inversed image $\mathcal{I}_{s i}$ in which hazy areas are rendered with enhanced contrast, while the unaltered areas appear as the inverse of the initial image. To identify the regions affected by 
haze, we compute the difference between the hue channels of the original image $\mathcal{I}$ and $\mathcal{I}_{s i}$, and threshold it using a predefined value $\tau$. The value of $\tau$ facilitates the selection of those pixels that present similar aspect in both the initial and the semi-inverse version. The results in this paper have been generated with the default value $\tau=10^{\circ}$. Only pixels that have a hue disparity below this threshold $\tau$ are labeled as hazy pixels. In our approach the hue information is represented by the $h^{*}$ channel after the image is transformed into the perceptual CIE $L^{*} c^{*} h^{*}$ color space.

By applying this simple strategy, we are able to estimate the hazy regions with acceptable precision. In order to check the validity of our observation, we collected a large database of natural images from several accessible photo sites (e.g. Flickr.com, Picasaweb.com, Photosig.com). Note that all the selected images have been taken in daily light conditions. We defined three main categories of outdoor images: haze-free images without sky, sky images, and hazy images. After manually selecting $800+$ images for each of these classes, we evaluated the variation of the hue using the strategy previously described. The main conclusion is that the haze-free images are characterized by a vast majority of pixels affected by significant hue variations, while in the other two categories this variation is considerably less. We illustrate this in Fig. 4 . In order to differentiate between the latter categories, it is possible to detect sky regions using existing techniques [17]. For a comparison of our haze detection component with the dark channel method of He et al. [3], we refer to the discussion section.

\section{Our Approach}

\subsection{Airlight Color $\left(A_{\infty}\right)$ Estimation}

One important correlation for dehazing algorithms constitutes the relation between optical depth and airlight $[18,11]$. The airlight $\mathcal{A}$ becomes more dominant as the optical depth increases. The optical model (equation 1) reveals the fact that two objects with different reflectance properties, located at the same distance from the observer, have identical airlight gains. Consequently, when observing the values of $\mathcal{A}$ in a small area around a scene point, they usually show only minor variations, except when depth discontinuities occur. Moreover, the $A_{\infty}$ constant can be acquired from the areas with the highest additive contribution, which are commonly the areas of the image characterized by high intensity.

These properties of the hazy images have been exploited as well in the previous approaches to estimate the airlight constant $A_{\infty}$. As observed by Narasimhan and Nayar [9], this constant is best estimated in the most haze-opaque areas. He et al. [3] choose the $0.1 \%$ brightest pixels of the dark channel as their preferred region. Another approach [2] is to search for this component in regions with the highest intensity, assuming that the sky is present and that there are no saturated pixels in the image.

The key advantage of our approach is that we are able to clearly identify hazy regions. As explained in section 4, these regions are identified in a straightforward manner by observing the hue disparity between the image and its semi-inverse. 

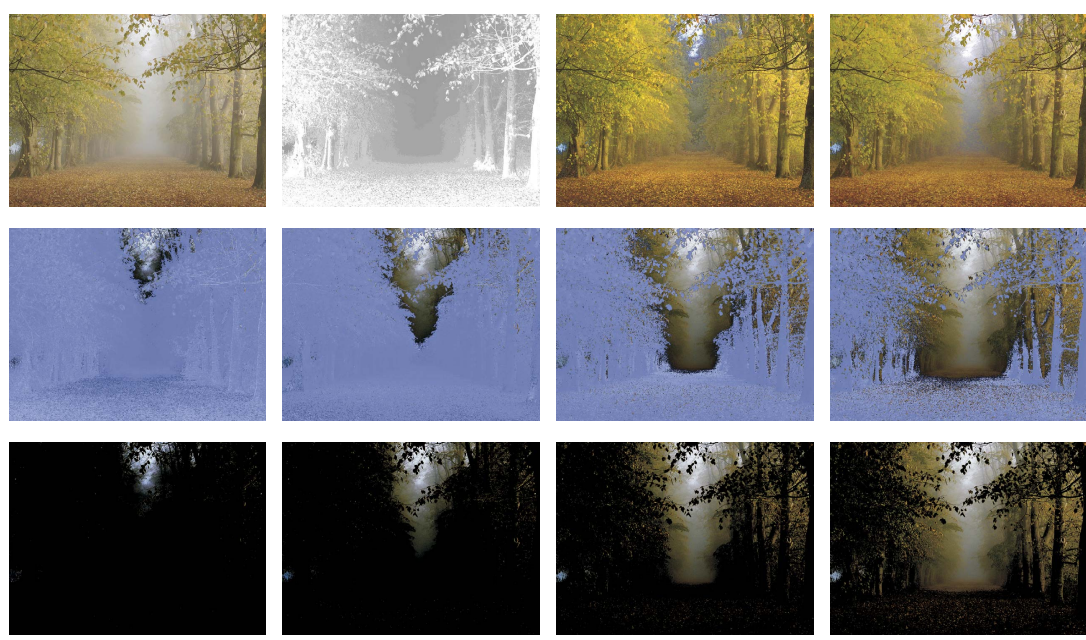

Fig. 5. Layer-based dehazing. Top line: the initial foggy image; the rough transmission map that corresponds to $\mathcal{I}_{0}$; the result of a naive method which simply pastes all layers $\mathcal{L}_{i}$ upon each other, introducing artifacts; the result of our method, which applies soft blending of the layers. Middle line: the mask regions for each layer. Bottom line: the computed layers $\mathcal{L}_{i}$.

In order to mask the most haze-opaque areas, we perform the same procedure, but with the intensity of the semi-inverse increased by a factor $\xi$ (with a default value of $\xi=0.3$ ). During our experiments, we found that for images where the sky is present, the resulted mask contains mostly the sky region, which decreases the searching space. The extraction of the airlight color vector $A_{\infty}$ is performed by determining the brightest pixel only in the positive (unmasked) region (see Fig. 3). The winning value of $A_{\infty}$ is extracted from the original foggy image from the same location as the brightest pixel. This approach has shown to be more robust than only searching for the most brightest pixels in the entire image.

\section{$5.2 \quad$ Layer-based Dehazing}

The contrast within an dehazed image is directly correlated with the estimated airlight $\mathcal{A}$ and the inferred transmission $t(x)$ at each point. Due to the physical additive property of the airlight (see equation 1), it is possible to estimate the direct attenuation $\mathcal{D}$ once the $A_{\infty}$ is known, by varying $\mathcal{A}=A_{\infty} *(1-t(x))$ in all possible values of its range [2]. Previous approaches have introduced many constraints and cost functions that favor certain image characteristics based on local image patches, thus limiting this range, and making it possible to compute an approximate transmission map. In previous strategies, the transmission map is commonly refined further using an energy minimization approach, based on the assumption that for local neighborhoods the airlight shows only very minor deviations (an assumption that breaks down at depth discontinuities). The main 

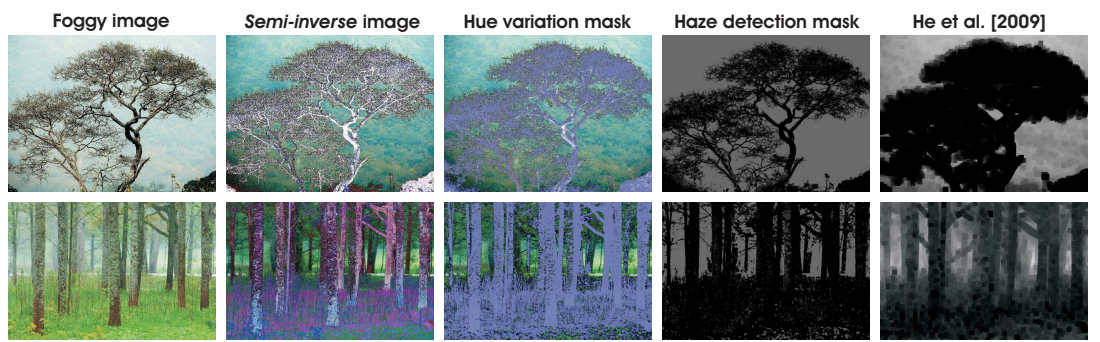

Fig. 6. Our technique is able to identify hazy regions on a per pixel basis. Comparing the haze mask produced by our technique to the dark channel mask of He et al. [3] clearly shows that we are able to preserve significantly more detail.

drawback of such approaches is that the employed search methods, even though they are commonly very expensive, are unable to ensure an accurate transmission map. In contrast, we present a fast method which segments the image in regions that do not contain abrupt depth discontinuities. Our strategy was inspired by the approach of Narasimhan and Nayar [9], which aims to normalize the pixels in so-called iso-depth neighborhoods. However, the previous method requires two pictures in order to identify such regions for their normalization operation. When using multiple images, it becomes possible to identify such isodepth neighborhoods, as they are invariant to the weather conditions and do not contain sudden depth discontinuities [9].

There are many possible strategies to create a dehazed image after a per pixel identification of hazy regions. In this work, we propose a layer-based method, which aims at preserving a maximum amount of detail, while still retaining sufficient speed. We initiate our algorithm by creating several new images $\mathcal{I}_{i}$, with $i \in[1, k]$ and $k$ layers, in which we remove a decreasingly growing portion of the airlight constant color $A_{\infty}$ from the initial hazy image $\mathcal{I}$ :

$$
\mathcal{I}_{i}=\mathcal{I}-c_{i} \cdot A_{\infty}
$$

with the iteratively increasing airlight contribution factor $c_{i}$. Parameter $c_{i}$ is a scalar value in the range $[0,1]$ with its value depending by the number of layers.

After applying our haze detection operation on $\mathcal{I}_{i}$, only the pixels with a sufficiently low hue disparity are labeled as being part of layer $\mathcal{L}_{i}$. In the absence of the scene geometry, discretization of the image in $k$ distinct layers enables us to estimate the values of $c_{i}$ that correspond to the most dominant depth layers of the scene. For instance, when the scene contains two objects located at different depths, the transmission map will be characterized by two dominant values (as the airlight is correlated with the distance). Finally, these layers are blended into a single composite haze-free image. In order to smooth the transitions between the different layers, the number of extracted layers $k$ needs to be at least 5 or more. As can be observed in Fig. 5 every layer (except the first one) includes the pixels of the previous layer, but with different levels of attenuation. The fast generated results in our paper have been yielded using a default value of 5 
layers and the values for $c_{i}:[0.2 ; 0.4 ; 0.6 ; 0.8 ; 1]$. To obtain the haze-free image $\mathcal{I}_{0}$, the layers are blended in the descending order of the airlight contribution. A naive approach would consist of simply copying the pixels from each layer on the next, but this might generate unpleasing artifacts due to small discontinuities (see Fig. 5). In order to remove such undesirable transitions artifacts, each layer will contribute a small percentage onto the next layer, according to the following equation:

$$
\mathcal{I}_{0}=\sum_{i=1}^{k} \chi_{i} \mathcal{L}_{i} .
$$

where the scalar parameter $\chi_{i}$ weights the contribution of the layers pixels, increasing exponentially according with the layer number. Splitting the input image into non-uniform neighborhoods that contains approximately uniform airlight generates good results, even when using only a single image. In comparison, algorithms that are based upon fixed-size patches may introduce artifacts because these uniform patches do not consider the intensity distribution of the small region. Moreover, our straightforward pixel based strategy is computationally effective overcoming the existing single image dehazing approaches (see the next section for comparative processing times).

\section{Discussion}

\subsection{Haze Detection}

As we have stated in the introduction, we believe to be the first to present an algorithm for per pixel haze detection. It could be stated that the algorithm of He et al. [3], an extension of the dark object technique common in the remote sensing community [6], could also be regarded as a method for haze area detection. However, in order to create the dark channel, their technique employs a patch-based approach which is unable to properly preserve any detail. When taking a closer look at Fig. 6, the patch-like structure of the dark channel images immediately becomes apparent. The black regions associated with haze-free areas do not reflect the true haze-free area borders. It is important to note that these transitions need to be recovered properly, as edges between two regions characterized by large illumination differences can generate prominent halo artifacts. This is the result of using patches with a constant dimension, which do not consider the intensity distribution of the small region. In constrast, our method employs a pixel-wise multi-layer strategy, which decomposes the image in regions that are characterized by small illumination transitions. This approach does not suffer from halo artifacts, because the regions are non-uniform and respect the image intensity distribution.

\subsection{Haze Removal}

We have tested our approach on a large data set of natural hazy images. Figure 7 illustrates results (our dehazed image and the computed transmission map) 


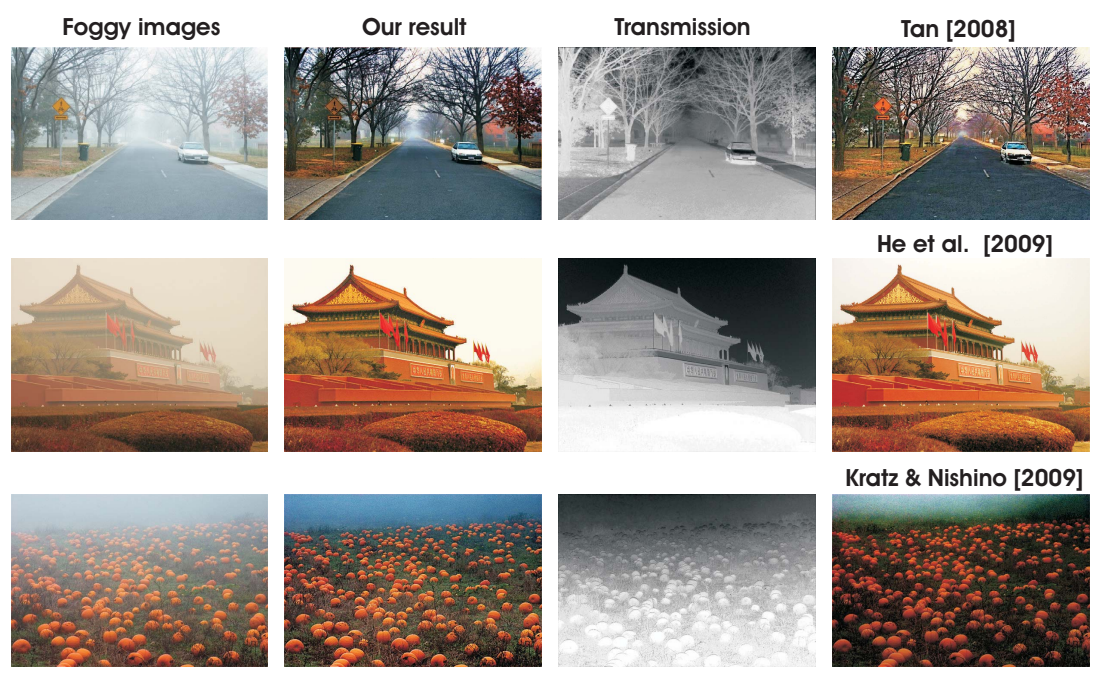

Fig. 7. Comparative haze removal results. From left to right: initial foggy images, our restored results, our estimated transmissions (depth map) and the corresponding results obtained by Tan [2], He et al. [3] and Kratz and Nishino [5] respectively.

obtained for three foggy images by our technique, compared to the methods of Tan [2],He et al. [3] and Kratz and Nishino [5]. All the figures presented in this paper and supplementary material contain the original restored images provided by the authors. As can be observed, we are able to enhance the images while retaining even very fine details. Furthermore, our method accurately preserves the color of the objects in the scene. Another set of images is provided in Fig. 9, where the top lines show comparative results obtained by the techniques of Fattal [1], He et al. [3], Tarel and Hautiere [4] and our method. It should be noted that in the discussion above, we have limited ourselves to images in which the scene is sufficiently illuminated. Even though the method performs generally well, for poorly lit scenes (an extreme case of such problem), like previous single image dehazing techniques, our approach may have trouble to accurately detect hazy regions. This limitation is due to the hypothesis of the considered optical model that assumes that regions characterized by small intensity variations contain no depth discontinuities.

As previously mentioned our approach has the advantage to perform faster than related algorithms. Our method implemented on CPU (Intel 2 Duo 2.00 $\mathrm{GHz}$ ) processes an $600 \times 800$ image in approximately 0.013 seconds being suitable for real-time outdoor applications. In comparison, the technique of Tarel and Hautiere [4] processes an $480 \times 600$ image in 0.2 seconds. However, as can be observed in Fig. 8 the patch-based method of Tarel and Hautiere [4] risks to introduce artifacts close to the patch transitions but also to distort the global contrast. The method of Tan [2] requires more than 5 minutes per image while the technique of Fattal [1] computes an $512 \times 512$ image in 35 seconds. The 

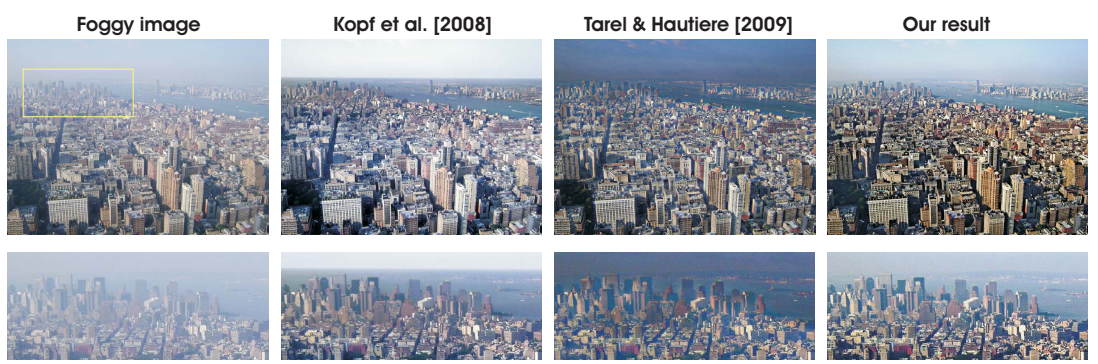

Fig. 8. From left to right: initial foggy image, the result obtained by Deep Photo [15] that employs additionally an approximated $3 \mathrm{D}$ model of the scene, the result of Tarel and Hautiere [4] and our result.

algorithm of He et al. [3] takes approximately 20 seconds per image while the computation times of the techniques of Kratz and Nishino [5] were not reported.

\subsection{Evaluating Dehazing Methods}

Since there is no specialized evaluation procedure of the dehazing techniques we searched the recent literature for an appropriate method for this task. Tarel and Hautiere [4] evaluate the quality of dehazing techniques based on a visibility resaturation procedure [19]. Because this procedure only applies to grayscale images and is mainly focused on finding the most visible edges, we searched for a more general method that is able to perform a pixelwise evaluation of the dehazing process.

In this work, we have employed the Image Quality Assessment (IQA) quality measure introduced recently by Aydin et al. [20], which compares images with radically different dynamic ranges. This metric, carefully calibrated and validated through perceptual experiments, evaluates both the contrast and the structural changes yielded by tone mapping operators. The IQA metric is sensitive to three types of structural changes: loss of visible contrast (green) - contrast that was visible in the reference image is lost in the transformed version, amplification of invisible contrast (blue) - contrast that was invisible in the reference image becomes visible in the transformed image, and reversal of visible contrast (red) - contrast visible in both images, but with different polarity. As a general interpretation, contrast loss (green) has been related with image blurring, while contrast amplification (blue) and reversal (red) have been connected to image sharpening.

Since these modifications are closely related to our problem, we have found this measure to be more appropriate as a means to evaluate the resaturation of hazy regions after applying different dehazing techniques.

Figure 9 shows the comparative results of applying the IQA metric on two foggy images and their dehazed versions, using the method of Fatal [1], He et al. [3], Tarel and Hautiere [4] and ours. The bottom-left table of Fig. 9 displays the comparative ratios of the (colored) pixels yielded by the IQA measure 


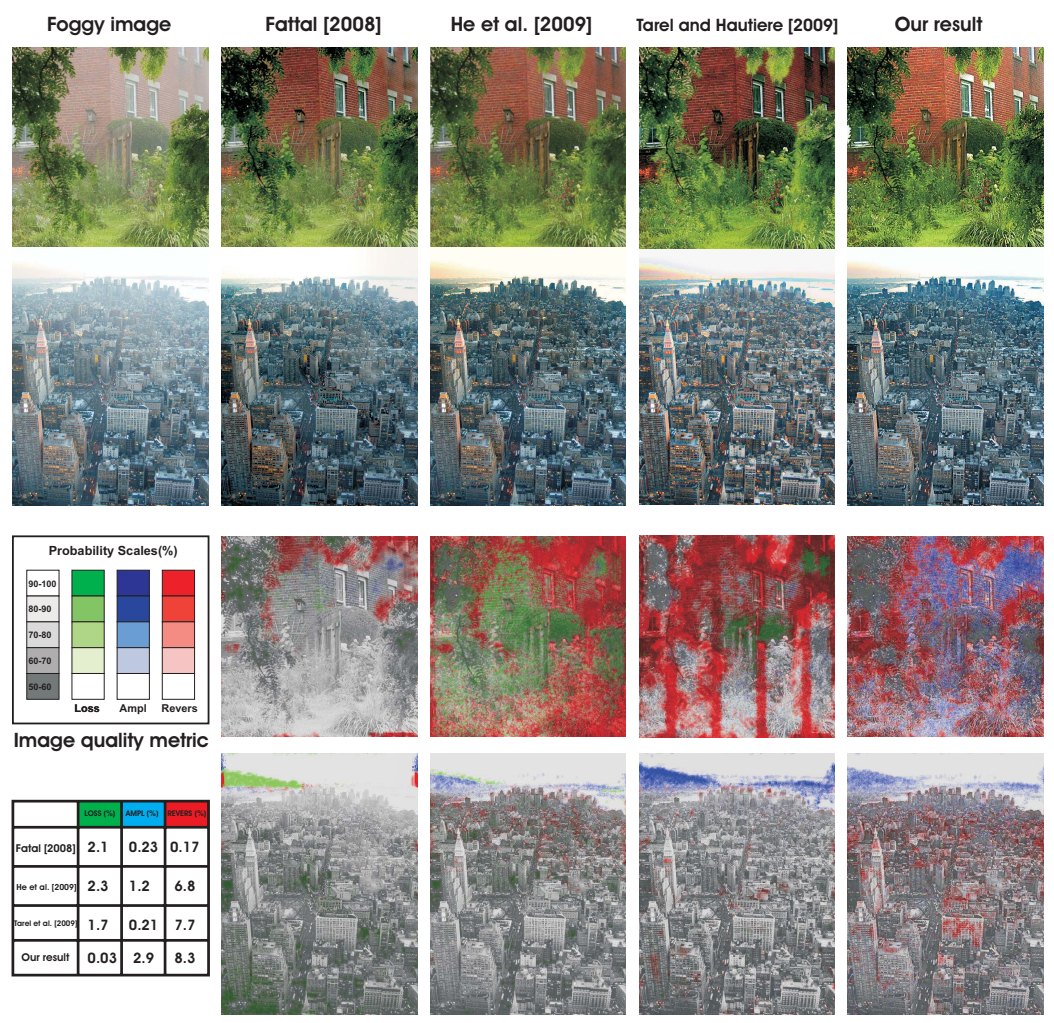

Fig. 9. Evaluation of the results using the IQA metric. The top two lines present the comparative results obtained by Fattal [1], He et al. [3], Tarel and Hautiere [4] and our method. The bottom two lines show the results after applying the IQA between initial image and the restored version. The left bottom table presents the ratio of the color pixels (with a probability scale higher than $70 \%$ ) counted for each method.

when applied to the results of the considered dehazing methods. Following the recommendation of Aydin et al. [20], in order to reduce the possibility of misclassification, only the pixels with a probability scale higher than $70 \%$ have been considered. Based on the results from the table, it becomes clear that compared with the other techniques, the structural changes yielded by our algorithm are more closely related to sharpening operations (blue and red pixels) and less related with blurring (green pixels).

\section{Conclusions}

In this work, we have presented a single-image dehazing strategy which does not make use of any additional information (e.g. images, hardware, or available depth information). Our approach is conceptually straightforward. Based on a per pixel hue disparity between the observed image and its semi-inverse, we are able to 
identify the hazy regions of the image. After we have identified these regions, we are able to produce a haze-free image using a layer-based approach. The processing time of our technique is very low, even when compared to previous methods who were designed and optimized for speed. In the future, we will be investigating a more comprehensive optical model, as well as extending our work to the problem of video dehazing.

\section{References}

1. Fattal, R.: Single image dehazing. SIGGRAPH, ACM Transactions on Graphics (2008)

2. Tan, R.T.: Visibility in bad weather from a single image. In IEEE Conference on Computer Vision and Pattern Recognition (2008)

3. He, K., Sun, J., Tang, X.: Single image haze removal using dark channel prior. In IEEE Conference on Computer Vision and Pattern Recognition (2009)

4. Tarel, J.P., Hautiere, N.: Fast visibility restoration from a single color or gray level image. In IEEE International Conference on Computer Vision (2009)

5. Kratz, L., Nishino, K.: Factorizing scene albedo and depth from a single foggy image. In: IEEE International Conference on Computer Vision. (2009)

6. Chavez, P.: An improved dark-object subtraction technique for atmospheric scattering correction of multispectral data. Remote Sensing of Environment (1988)

7. Moro, G.D., Halounova, L.: Haze removal and data calibration for high-resolution satellite data. Int. Journal of Remote Sensing (2006)

8. Narasimhan, S., Nayar, S.: Chromatic Framework for Vision in Bad Weather. In: In IEEE Conference on Computer Vision and Pattern Recognition. (2000)

9. Narasimhan, S., Nayar, S.: Contrast Restoration of Weather Degraded Images. IEEE Trans. on Pattern Analysis and Machine Intell. (2003)

10. Schaul, L., Fredembach, C., Ssstrunk, S.: Color image dehazing using the nearinfrared. In IEEE Int. Conf. on Image Processing (2009)

11. Treibitz, T., Schechner, Y.Y.: Polarization: Beneficial for visibility enhancement? In IEEE Conference on Computer Vision and Pattern Recognition (2009)

12. Shwartz, S., Namer, E., Schechner, Y.: Blind haze separation. In IEEE Conference on Computer Vision and Pattern Recognition (2006)

13. Namer, E., Shwartz, S., Schechner, Y.: Skyless polarimetric calibration and visibility enhancement. Optic Express (2009) 472-493

14. Narasimhan, S., Nayar, S.: Interactive de-wheathering of an image using physical models. ICCV Workshop CPMVC (2003)

15. Kopf, J., Neubert, B., Chen, B., Cohen, M., Cohen-Or, D., Deussen, O., Uyttendaele, M., Lischinski, D.: Deep photo- Model-based photograph enhancement and viewing. In: ACM Transactions on Graphics. (2008)

16. Koschmieder, H.: Theorie der horizontalen sichtweite. In: Beitrage zur Physik der freien Atmosphare. (1924)

17. Tao, L., Yuan, L., Sun, J.: SkyFinder: Attribute-based Sky Image Search. SIGGRAPH, ACM Transactions on Graphics (2009)

18. R.C.Henry, S.Mahadev, Urquijo, S., Chitwood, D.: Color perception through atmospheric haze. Opt. Soc. Amer. A 17 (2000) 831-835

19. Hautiere, N., Tarel, J.P., Aubert, D., Dumont, E.: Blind contrast enhancement assessment by gradient ratioing at visible edges. Img. Anal. and Stereology (2008)

20. Aydin, T.O., Mantiuk, R., Myszkowski, K., Seidel, H.S.: Dynamic range independent image quality assessment. In: SIGGRAPH, ACM Trans. Graph. (2008) 